\title{
On Wheeler's delayed-choice Gedankenexperiment and its laboratory realization
}

\author{
M Božići ${ }^{1}$ L Vušković ${ }^{2}, \mathbf{M}$ Davidović ${ }^{3}$ and A S Sanz ${ }^{4}$ \\ ${ }^{1}$ Institute of Physics, University of Belgrade, 11080 Belgrade, Serbia \\ ${ }^{2}$ Department of Physics, Old Dominion University, Norfolk, Virginia, USA \\ ${ }^{3}$ Faculty of Civil Engineering, University of Belgrade, 11000 Belgrade, Serbia \\ ${ }^{4}$ Instituto de Física Fundamental - CSIC, Serrano 123, 28006 - Madrid, Spain \\ E-mail: bozic@ipb.ac.rs,vuskovic@odu.edu, milena@grf.rs,asanz@iff.csic.es
}

\begin{abstract}
Here, we present an analysis and interpretation of the experiment performed by Jacques et al. (2007 Science $\mathbf{3 1 5}$ 966), which represents a realization of Wheeler's delayed-choice Gedankenexperiment. Our analysis is based on the evolution of the photon state, since the photon enters into the Mach-Zehnder interferometer with a removable beam-splitter until it exits. Given the same incident photon state onto the output beam-splitter, $B S_{\text {output }}$, the photon's state at the exit will be very different depending on whether $B S_{\text {output }}$ is on or off. Hence, the statistics of photon counts collected by the two detectors, positioned along orthogonal directions at the exit of the interferometer, is also going to be very different in either case. Therefore, it is not that the choice of inserting (on) or removing (off) a beam-splitter leads to a delayed influence on the photon behavior before arriving at the beam-splitter, but that such a choice influences the photon state at and after $B S_{\text {output }}$, i.e., after it has exited from the MachZehnder interferometer. The random on/off choice at $B S_{\text {output }}$ has no delayed effect on the photon to behave as a wave or a corpuscle at the entrance and inside the interferometer, but influences the subsequent evolution of the photon state incident onto $B S_{\text {output }}$.
\end{abstract}

PACS numbers: 03.65.Ta, 42.50.Xa, 03.75.Dg, 37.25.+K

\section{Introduction}

Since the inception of Quantum Mechanics, various Gedankenexperimente were proposed, which made evident properties very different to those described by Classical Mechanics. With time, the necessity to test these fundamental properties led, in many cases, to the development of the technology necessary to pass from mere ideas to real experiments with real particles - either massive particles or photons.

One of such experiments is the well-known Wheeler's delayed-choice Gedankenexperiment [1], proposed to test the nature of wave or corpuscle of quantum particles by means of a Mach-Zehnder interferometer (MZI). In order to select one or the other behavior, the interferometer has a removable (output) beam-splitter at the exit (see Fig. 11). When the output beam-splitter is positioned on its place, the MZI configuration is said to be closed; when it is off place, the configuration is open. Following Wheeler's argument, with the first configuration one observes the wave behavior of the particle, while with the latter, the corpuscular one. 


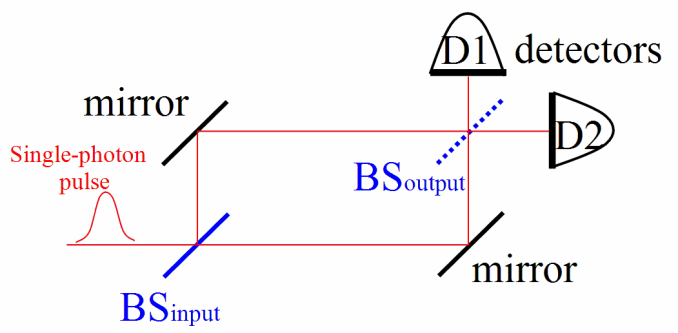

Figure 1. Schematics of an MZI with a removable beam-splitter at the output, as considered by Wheeler [1].

In 2007, Jacques et al. [2] carried out in the lab Wheeler's experiment. In this experiment, the choice between the open and closed configurations was realized by means of an electro-optical modulator, which could be switched at will between the two different configurations in times of the order of $40 \mathrm{~ns}$. This time is enough to close or to open the MZI configuration once the photon is inside. Furthermore, with this distance in time, the switching of the output beam-splitter and the entry of the photon into the MZI are events well separated in time relativistically. It is crucial there is no correlation in time between both events, which have to take place once the photon is inside the interferometer, as argued by Wheeler [1]. Otherwise the photon might acquire some "hidden information" on the chosen experimental configuration and could readjust its behavior accordingly. This was precisely the main reason leading Wheeler to formulate his experiment against other alternative experiments proposed at the time to test complementarity.

\section{Laboratory realization and looking backward interpretation}

In the experiment [2], single photons are sent towards a 48-m polarization interferometer, equivalent to a time-of-flight of about 160 ns. A binary random number, 0 or 1 , generated by a quantum random number generator (QRNG), drives the electro-optical-modulator (EOM) voltage between $V=0$ and $V=V_{\pi}$ within 40 ns, after an electronic delay of $80 \mathrm{~ns}$. Two synchronized signals from a clock are used to trigger the single-photon emission and the QNRG. Thus, the random choice between the open and closed MZI configurations takes place when the photon is approximately about the central part of the interferometer, long after it passed through $B S_{\text {output }}$. Moreover, a phase shift, $\phi$, between the two MZI arms is introduced by executing a tilt with a $B S_{\text {output }}$ piezoelectric actuator (PZT).

The chosen configuration, the detection events (which detector registered the event), and the PZT position were then recorded for each photon. All raw data were saved in the real time. For each PZT-position (phase), detection events at D1 and $D 2$ corresponding to each configuration were sorted out. Thus, when analyzing these data, one observes [2]:

A) The counts at $D 1$ and $D 2$ display sinusoidal oscillations with for the closed configuration (see Fig. 3A in Ref. [2]).

B) The counts at $D 1$ and $D 2$ do not depend on $\phi$ for the open configuration (see Fig. 3B in Ref. [2]). 
From these experimental results, Jacques et al. concluded [2] that "the behavior of the photon at the first beam splitter depends on the choice of the observable that is measured behind the output beam splitter, even when that choice is made at a position and a time such that it is separated from the entrance of the photon into the interferometer by a space-like interval", which according to Wheeler [1] translates as "a strange inversion of the normal order of time."

\section{Looking forward interpretation of the experiment}

Here, we analyze and propose an the intuitive interpretation of the experiment of Jacques et al. [2] by considering the evolution of the photon state from its entrance into the MZI and throughout its passage.

Beam-splitters are essential constituting elements of a MZI. In considering the action of a beam-splitter on a quantum object, it is fundamental to take into account the incident quantum state of such an object as well as the subsequent evolution of this state [3-8]. In this sense, a beam-splitter can be considered as a transformer of an incident wave field (photon field or matter wave field) into a field which has narrow maxima at the points along and in close vicinity of two or several specific directions. This becomes evident when one considers a thin grating as a model for a beam splitter for photons [3-5], atoms and molecules [6]. From such considerations, it follows that a lossless beam-splitter can also be termed as a coherent beam-splitter [7], since the outgoing "separated beams" do not spread independently, but jointly, keeping their mutual coherence.

Taking this into account, we note the following:

i) The input and output beam-splitters (in the latter case, when it is on) transform two different states of a photon. Therefore, the probabilities associated with the photon going through one or the other characteristic direction (of the two possible) behind $B S_{\text {input }}$ and $B S_{\text {output }}$ are different.

ii) The state of the photon incident onto $B S_{\text {output }}$ is determined by the interaction with $B S_{\text {input }}$, the two mirrors and the free-evolution equation. Therefore, the photon state incident onto $B S_{\text {output }}$ is independent of whether $B S_{\text {output }}$ is on or off.

iii) The evolution of a given photon state incident onto $B S_{\text {output }}$ depends on whether $B S_{\text {output }}$ is on or off. Therefore, the photon state at the exit of $B S_{\text {output }}$ when the latter is on is very different from the photon state when this beam-splitter is off. The statistics measured by the detectors $D 1$ and $D 2$ when $B S_{\text {output }}$ is off is then different from the statistics when it is on, because the state of the outgoing photon depends on the on/off state of $B S_{\text {output }}$.

When $B S_{\text {output }}$ is on, it changes the incidence photon state, which then evolves freely outside the interferometer. The probability that a photon chooses one or the other direction is determined by its incident state and the interaction with a grating. When the beam-splitter is off, the incidence photon state (as well as the photon itself) propagates freely. Consequently, a photon keeps moving through the direction along which it was moving before reaching $B S_{\text {output }}$.

The on/off switching of $B S_{\text {output }}$ does not influence the behavior of the photon before it has arrived to this beam-splitter. Such a switching influences the photon state both at $B S_{\text {output }}$ and at the exit from this beam-splitter. Therefore, the photon statistics at the detectors will also be influenced by the switching. 


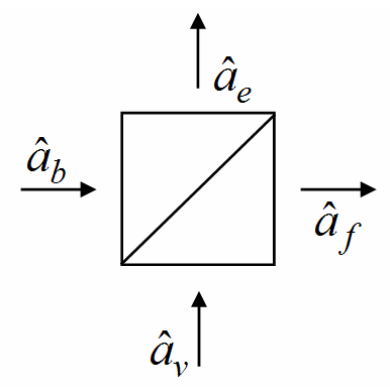

Figure 2. Schematic representation of the modes describing a beam-splitter.

So, we conclude that there is no delayed-choice action. The on/off switching does not decide whether the photon will move along one or both routes after it has already completed its travel; the on/off switching does influence the evolution of the photon state incident onto $B S_{\text {output }}$. In our opinion, the experiment of Jacques et al. [2] proves that the wave and corpuscle properties of photons are compatible, i.e., both are present in the same experiment.

\section{Mode operators and photon statistics at the exit of the MZI in the open and closed configurations}

The above conclusions can be alternatively derived in an elegant manner by considering a second-quantization treatment of the photon electromagnetic field in the MZI. To do so, note that the relationship between the input and output modes of the electromagnetic field surrounding a beam-splitter (see Fig. 22) are now well known [8]:

$$
\hat{a}_{s}=R \hat{a}_{b}+T \hat{a}_{v}, \quad \hat{a}_{f}=T \hat{a}_{b}+R \hat{a}_{v},
$$

The complex transmission and reflection coefficients for a lossless beam-splitter satisfy the relations

$$
\begin{aligned}
& R T^{*}+T R^{*}=0, \\
& |R|^{2}+|T|^{2}=1 .
\end{aligned}
$$

As can be seen in Fig. 3, the MZI closed configuration consists of two beam-splitters and two mirrors. The second beam-splitter may be tilted in order to introduce a phase shift. Thus, three sets of modes are necessary to describe the photon states in the MZI, namely $\left(\hat{a}_{b}, \hat{a}_{v}\right),\left(\hat{a}_{e}, \hat{a}_{f}\right)$ and $\left(\hat{a}_{c, 1}, \hat{a}_{c, 2}\right)$.

The relations between the output modes, $\left(\hat{a}_{c, 1}, \hat{a}_{c, 2}\right)$, and the internal ones, $\left(\hat{a}_{e}, \hat{a}_{f}\right)$, are similar to relations (1), but containing the additional phase shifts $\phi_{e}$ and $\phi_{f}$ in order to account for the tilting of $B S_{\text {output }}$,

$$
\begin{aligned}
& \hat{a}_{c, 1}=R \hat{a}_{e} e^{i \phi_{e}}+T \hat{a}_{f} e^{i \phi_{f}}, \\
& \hat{a}_{c, 2}=R \hat{a}_{f} e^{i \phi_{f}}+T \hat{a}_{e} e^{i \phi_{e}} .
\end{aligned}
$$

Now, using relations (11) one can determine the relationship between the output and input modes, which reads as [8]

$$
\begin{aligned}
& \hat{a}_{c, 1}=R_{M Z} \hat{a}_{b}+T_{M Z} \hat{a}_{v}, \\
& \hat{a}_{c, 2}=T_{M Z} \hat{a}_{b}+R_{M Z}^{\prime} \hat{a}_{v},
\end{aligned}
$$




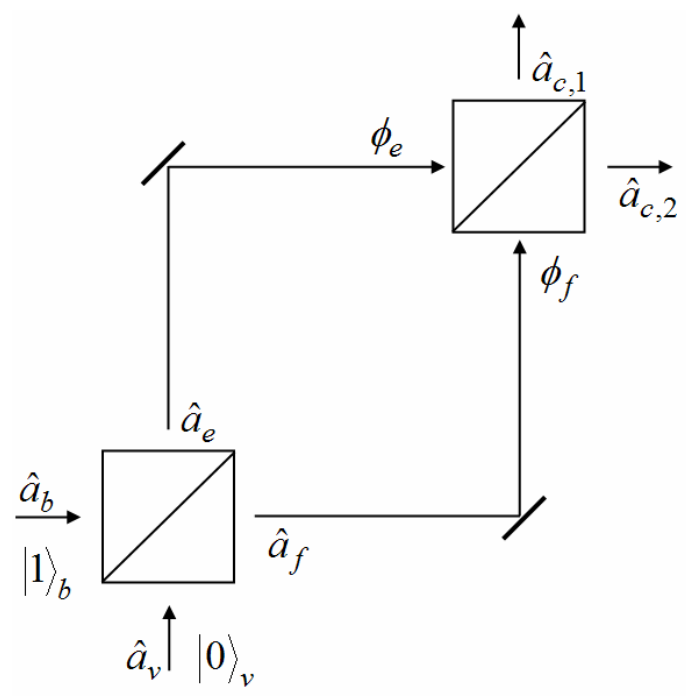

Figure 3. Schematic representation of the modes describing the MZI closed configuration, where $B S_{\text {output }}$ is on.

where

$$
\begin{aligned}
& R_{M Z}=R^{2} e^{i \phi_{e}}+T^{2} e^{i \phi_{f}}, \\
& R_{M Z}^{\prime}=R^{2} e^{i \phi_{f}}+T^{2} e^{i \phi_{e}}, \\
& T_{M Z}=R T\left(e^{i \phi_{e}}+e^{i \phi_{f}}\right) .
\end{aligned}
$$

In the open configuration of the MZI (see Fig. 4), input and internal modes are the same as in the closed configuration: $\left(\hat{a}_{b}, \hat{a}_{v}\right),\left(\hat{a}_{e}, \hat{a}_{f}\right)$. We shall denote the output modes by $\left(\hat{a}_{o, 1}, \hat{a}_{o, 2}\right)$. Since the output beam-splitter is off, the relations between output and input modes in the MZI open configuration are:

$$
\hat{a}_{o, 1}=T \hat{a}_{b}+R \hat{a}_{v}, \quad \hat{a}_{o, 2}=R \hat{a}_{b}+T \hat{a}_{v},
$$

Determining now the photon statistics behind the MZI in the open and closed configurations is straightforward. In the open configuration, the mean photon numbers at the detectors will be

$$
\begin{aligned}
& \frac{N_{o, 1}}{N}=\left\langle\hat{n}_{o, 1}\right\rangle={ }_{v}\left\langle\left. 0\right|_{b}\left\langle 1\left|\hat{a}_{o, 1}^{+} \hat{a}_{o, 1}\right| 1\right\rangle_{b} \mid 0\right\rangle_{v}=|T|^{2}, \\
& \frac{N_{o, 2}}{N}=\left\langle\hat{n}_{o, 2}\right\rangle={ }_{v}\left\langle\left. 0\right|_{b}\left\langle 1\left|\hat{a}_{o, 2}^{+} \hat{a}_{o, 2}\right| 1\right\rangle_{b} \mid 0\right\rangle_{v}=|R|^{2},
\end{aligned}
$$

with $N$ being the total number of incident photons. Therefore, the numbers of photons that propagate towards detectors $D 1$ and $D 2$ do not depend on the phase $\phi$, which is in agreement with the experiment. This is simple to understand. If the beam-splitter is off, there is no way its tilt can influence the motion and passage state of a photon. On the other hand, in the closed configuration, the number of photons at the detectors will be

$$
\begin{aligned}
\frac{N_{c, 1}}{N} & =\left\langle\hat{n}_{c, 1}\right\rangle={ }_{v}\left\langle\left. 0\right|_{b}\left\langle 1\left|\hat{a}_{c, 1}^{+} \hat{a}_{c, 1}\right| 1\right\rangle_{b} \mid 0\right\rangle_{v}=\left|R_{M Z}\right|^{2} \\
& =|R|^{4}+|T|^{4}-2|R|^{2}|T|^{2} \cos \phi, \\
\frac{N_{c, 2}}{N} & =\left\langle\hat{n}_{c, 2}\right\rangle={ }_{v}\left\langle\left. 0\right|_{b}\left\langle 1\left|\hat{a}_{c, 2}^{+} \hat{a}_{c, 2}\right| 1\right\rangle_{b} \mid 0\right\rangle_{v}=\left|T_{M Z}\right|^{2} \\
& =2|R|^{2}|T|^{2}(1-\cos \phi),
\end{aligned}
$$




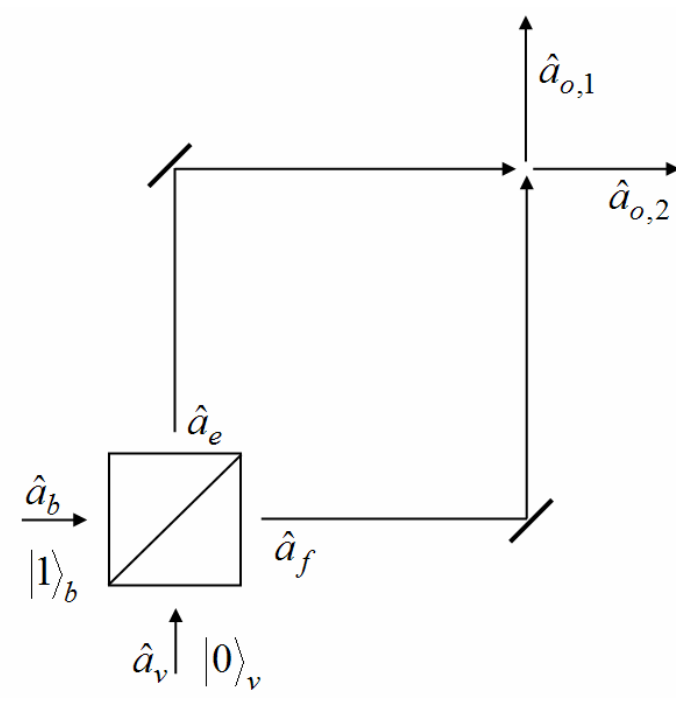

Figure 4. Schematic representation of the modes describing the MZI open configuration, where $B S_{\text {output }}$ is off.

where

$$
\phi \equiv \phi_{e}-\phi_{f}
$$

In deriving Eq. 8, the moduli and phases of the complex coefficients $R$ and $T$ were introduced taking into account relations 2 ,

$$
R=|R| e^{i \varphi_{R}}, \quad T=|T| e^{i \varphi_{T}}, \quad \varphi_{R}-\varphi_{T}=\frac{\pi}{2},
$$

Assuming $|R|=|T|=1 / \sqrt{2}$, one finds simpler relations for the number of photons along the two directions in the closed configuration:

$$
\begin{aligned}
& N_{c, 1}=\frac{N}{2}(1-\cos \phi), \\
& N_{c, 2}=\frac{N}{2}(1+\cos \phi) .
\end{aligned}
$$

\section{Comparison of the arguments leading to the two different interpretations}

The reasoning leading to the conclusion that particle properties are complementary $[1,2]$ and that "we have a strange inversion of the normal order of time" $[1,2]$ were based on the following two statements:

1) When $B S_{\text {output }}$ is off, the number of detected photons $N_{0,1}$ at the detector $D 1$ is equal to the number of detected photons $N_{0,2}$ at $D 2$. In this case, we have $N_{0,1}=N_{0,2}=N / 2$, which does not depend on the phase $\phi$. Hence, one measures the corpuscle property associated with the photon.

2) When $B S_{\text {output }}$ is on, the number of photons detected, $N_{c, 1}$ and $N_{c, 2}$, depend on the phase $\phi$, i.e., $N_{c, 1}=N_{c, 1}(\phi)$ and $N_{c, 2}=N_{c, 2}(\phi)$. In this case, therefore, one measures a wave property of the photon. 
This reasoning is based on the statement that the measurement of the same quantity, namely number of photons, some times has the meaning of a particle property measurement and other times it acquires the meaning of a wave property measurement.

On the contrary, the reasoning leading to the conclusion that particle and wave properties are compatible is based on the statement that the evolution of the same photon state incident onto the output beam splitter depends on whether this beam splitter is on or off. As a consequence, the relations between creation and annihilation operators associated with the input and output modes are different in the on and off cases:

1) When the beam splitter is off, the wave function of each single photon incident onto $B S_{\text {output }}$ evolves freely. Because of that, the number of photons at the detectors is determined by (7), i.e., it does not depend on the phase. The numbers at the detectors are equal to the numbers of photons arriving from the corresponding directions to $B S_{\text {output }}$.

2) When $B S_{\text {output }}$ is on, it influences the wave function evolution of each arriving photon. Consequently, the number of photons moving at the exit towards one or the other detector is changed, depending of the specific property of $B S_{\text {output }}$, e.g., its tilt, which reflects in the phase.

The reasoning that takes into account the wave function of each photon for both cases, on and off, leads to the consistent explanation of why the number of photons at the detectors is constant when $B S_{\text {output }}$ is off and varies with the tilt when it is on. It means that wave and particle properties of the photon are present simultaneously in the open and closed configurations. Thus, the time-ordering of events arises as a natural consequence within this reasoning, i.e., there is no "strange inversion of the normal order of time".

\section{Acknowledgments}

M. Božić and M. Davidović acknowledge support from the Ministry of Science of Serbia through projects III45016 and OI171005. A. S. Sanz acknowledges the Ministerio de Ciencia e Innovación (Spain) for support through projects FIS2007-62006 and FIS2010-22082 as well as for a "Ramón y Cajal" Research Contract.

\section{References}

[1] Wheeler J A 1978 The "past" and the "delayed-choice" double-slit experiment Mathematical Foundations of Quantum Mechanics ed A R Marlow (New York: Academic Press) pp 9-48

[2] Jacques V, Wu E, Grosshans F, Treussart F, Grangier P, Aspect A and Roch J-F 2007 Science 315966.

[3] Goulielmakis E, Nersisyan G, Papadogiannis N A, Charalambidis D, Tsakiris G D and Witte K 2002 Appl. Phys. B $\mathbf{7 4} 197$

[4] Davidović M, Sanz A S, Arsenović D, Božić M and Miret Artés S 2009 Phys. Scr. T135 014009

[5] Sanz A S, Davidović M, Božić M and Miret-Artés M 2010 Ann. Phys. 325763

[6] Arsenović D, Božić M and Vušković L 2002 J. Opt. B: Quantum Semiclass. Opt. 4 S358

[7] Božić M, Dimić D and Davidović M 2009 Acta Phys. Polonica A 116479

[8] Loudon R 2004 The Quantum Theory of Light (Oxford: Oxford University Press) 3rd Ed 